\title{
V367 Cyg as the interacting binary
}

\author{
H.Schneider*, Universitäts-Sternwarte, Göttingen, F.R.G. \\ K.Pavlovski, Hvar Observatory, Zagreb, Yugoslavia \\ M.C.Akan, Ege University Observatory, Izmir, Turkey
}

(Not reviewed)

Abstract: The interacting binary V367 Cyg is contained in the original list of W Serpentis stars discovered by Plavec and Koch (1978) in their initial UV study and has been subject of numerous studies, both photometric and spectroscopic.

A reasonable model of the system shows the primary as a supergiant of type A7 with $T_{\text {eff }}=8000^{\circ} \mathrm{K}, R=50 R_{\odot}$ and $\log g=1.5$, the secondary as A0III with $T_{\text {eff }}=10000^{\circ} \mathrm{K}$ and $R=7 R_{\odot}$. A cooler disk with a radius of $\approx 25 R_{\odot}$ encircles the secondary and the whole system is surrounded by a gasous shell of $\approx 500 R_{\odot}$.

But there is still a considerable confusion regarding the very basic geometric and radiative properties of V367 Cyg. Therefore we have analysed the available photometric data in the framework of the semi-detached configuration which incorporates the influence of an optically thick disk around the mass gaining component. The input data have been selected from the most recent spectroscopic studies based on the optical and UV spectra.

Our trial calculations show that a solution is possible in the framework of the assumed model. In our solution the disk around the gainer is in contact with the mass-loosing component and the radiative properties mimic a stellar atmosphere. This explains previous light curve solutions in terms of a contact configuration. Also, our model corrobates with a description of the W Serpentis stars as strongly interacting binaries in or near the phase of rapid mass transfer.

In August 1988 we could record eight CCD spectra of the $H \gamma$ region with a resolution of $0.13 \AA / p x$ taken during the primary eclipse (phase $0.692-0.163$ ). The spectra have been obtained at the German-Spanish Observatory at Calar Alto/Spain with the $2.2 \mathrm{~m}$ telescope and the Coudé spectrograph.

The $H_{\gamma}$ profiles display a very complex structure with at least four emission components. In the primary minimum the main emission components are of nearly the same intensity. It means, that we observe the outermost parts of the disk even during the eclipse. This is in agreement with the light curve solution.

The central depth of the shell lines changes only slightly, because they belong to the circumbinary shell. Besides this, some of the shell lines show broad red- or blue-shifted wings (depending on the orbital phase). They are probably originated from the spectrum of the primary.

References:

Plaves, W.,Koch,R.H., 1978: IBVS No.1482

* Visiting Astronomer, German-Spanish Astronomical Center, Calar Alto, operated by the Max-PlanckInstitut für Astronomje Heidelberg jointly with the Spanish National Commision for Astronomy. 